\title{
RATES OF EVOLUTION AND HOMOPLASY
}

HAUSER, David L., Department of Biology, University of Pennsylvania, Philadelphia PA 19104; BOYAJIAN, George E, Department of Geology, University of Pennsylvania, Philadelphia PA 19104; SHUBIN, N.H., Department of Biology, University of Pennsylvania, Philadelphia PA 19104

Homoplasy is present in virtually every known systematic analysis and exists at all levels of the Linnaean hierarchy. Despite the nearly universal presence of homoplasy, the patterns and causal mechanisms of it have only recently been addressed.

Sanderson and Donoghue (1989) examined the relationships between homoplasy (as measured by the consistency index) and taxonomic rank, the number of characters, and the number of taxa. Their analysis, which involved 60 data sets from the literature, showed a statistically significant and inverse correlation between the number of taxa and the amount of homoplasy exhibited.

In order to expand on Sanderson and Donoghue's important contribution, we attempt to dissect the causal mechanisms of homoplasy. As a working hypothesis, we assume that if the number of character states is limited, homoplasy should be a function of at least three factors: 1) the number of cladogenetic events (which is directly related to the number of taxa), 2) the percentage of characters that undergo change at each node, and 3) the probability of any one character changing at any one node. Because the absolute, or even relative, values of the last two factors are rarely known for a given taxonomic group, we used computer generated data sets in order to quantitatively determine the relative importance of these three factors to patterns of homoplasy. Characters were allowed to "evolve" on a preset tree topology; this process was repeated for a wide variety of values for each of the above factors. The correlation of each factor with the consistency index was then examined. In order to control for results that were unique to a given tree topology, the same data sets were generated onto two very different tree topologies (a extensively bifurcated tree and a Hennigian comb).

Our preliminary results indicate that homoplasy is much more sensitive to the percentage of characters that change at a given node then to the number of taxa involved (as determined by the amount of variance explained by a regression line). In addition, the nature of the above procedure allows for the analysis of the dynamic relationship between evolutionary rate and constraint (defined as a limitation in the number of character states possible). These two factors conspire to produce homoplasy. The effect of high rates of evolution on homoplasy is magnified by successively greater constraints on character states; conversely, high rates of evolution in conjunction with low degrees of constraint lead to lesser levels of homoplasy. 